\title{
Positive Association of Male Overactive Bladder Symptoms and Androgen Deprivation: A Nationwide Population-based Cohort Study
}

\author{
JIAN-RI LI I,2,3, SHIAN-SHIANG WANG ${ }^{1,2}$, CHING-HENG LIN ${ }^{4}$, \\ WILLIAM C. DE GROAT ${ }^{5}$ and CHEN-LI CHENG ${ }^{1,2}$ \\ ${ }^{1}$ Division of Urology, Department of Surgery, Taichung Veterans General Hospital, Taichung, Taiwan, R.O.C.; \\ ${ }^{2}$ Institute of Medicine, Chung Shan Medical University, Taichung, Taiwan, R.O.C.; \\ ${ }^{3}$ Department of Medicine and Nursing, Hungkuang University, Taichung, Taiwan, R.O.C.; \\ ${ }^{4}$ Department of Medical Research, Taichung Veterans General Hospital, Taichung, Taiwan, R.O.C.; \\ ${ }^{5}$ Department of Pharmacology and Chemical Biology, \\ University of Pittsburgh School of Medicine, Pittsburgh, PA, U.S.A.
}

\begin{abstract}
Background/Aim: The role of androgen in the modulation of voiding function is still uncertain. The aim of this study was to evaluate the association of androgen deprivation therapy $(A D T)$ and overactive bladder $(O A B)$ in men in a population-based cohort. Materials and Methods: This study examined the records of newly-diagnosed prostate cancer subjects receiving ADT only in the Taiwan National Health Insurance Research Database in the years between 2001 and 2007. As controls men without cancer were selected and divided into three groups, 1) benign prostate hyperplasia treated with an alpha-blocker (BPH-alpha blocker), 2) BPH treated with a 5alpha reductase inhibitor (BPH-5ARI) and 3) healthy controls. $O A B$ events were censored by definition of drug prescriptions for more than one month and risk analysis among each group was performed. Results: The healthy control group had decreased risk of $O A B$ compared to the prostate cancer group and the BPH-5ARI group showed a higher risk of OAB than the prostate cancer group. Subgroup analysis showed that independently of age or comorbidities, the prevalence of $O A B$ was significantly lower in the healthy control group. Moreover, the cumulative incidence of $O A B$ showed a time-dependent pattern with a significant increase after ADT for 5 years. Conclusion: Androgen deprivation in prostate cancer patients
\end{abstract}

Correspondence to: Chen-Li Cheng, MD, Ph.D., Division of Urology, Department of Surgery, Taichung Veterans General Hospital, 1650 Sec. 4, Taiwan Blvd, Taichung, Taiwan, R.O.C. Tel: +886 423592525 ext 5006, Fax: +886 423593160, e-mail: Cheng20011@gmail.com

Key Words: Androgen, benign prostate hyperplasia, overactive bladder, prostate cancer. was associated with an increased risk of $O A B$ that was treatment duration-dependent. This result is consistent with an inhibitory role of androgen in the modulation of male voiding function.

Sex hormones including androgen and estrogen bind to steroid receptors that are widely distributed in human tissues $(1,2)$. In genitourinary tract, sex hormones not only control the appearance and function of sex organs, but may also influence lower urinary tract function (3). Our previous human population cohort studies as well as experimental studies on female rats suggested that estrogen may influence the voiding function in females $(4,5)$. However, the role of androgen in the modulation of male voiding functions is less certain. Epidemiological studies showed that aging which is accompanied by decreased serum testosterone levels $(6,7)$ is also associated with increased prevalence of overactive bladder $(\mathrm{OAB})$. We also showed that orchiectomy decreased the volume threshold for inducing micturition in a rat model (8). Based on current evidence, serum testosterone seems to play a role in the modulation of voiding function in men (9). However, a relationship between serum testosterone level and OAB has not been established.

In this study, a nationwide population-based insurance database was used to examine a possible link between androgen deprivation therapy (ADT) and $\mathrm{OAB}$ in prostate cancer patients. The data were compared with data obtained from healthy controls and from patients with benign prostate hyperplasia (BPH).

\section{Materials and Methods}

Data sources. All data in this study were obtained from the Taiwan National Health Insurance Research Database (NHIRD), which is managed by the Taiwan National Health Research Institute (NHRI). 
The Taiwan National Health Insurance (NHI) program covers at least $99 \%$ of the population of the island since 1995. In this study, a systemic sampling from the Taiwan NHIRD between 2001 and 2007 with a total of $1,000,000$ individuals was used. These random subjects have been approved by the NHRI to be representative of the general population in Taiwan. The study protocols were approved by the institutional review board (IRB) of Taichung Veterans General Hospital (CE13151-1) and were in accordance with the guidelines of the IRB. There were no statistically significant differences in age and general healthcare between the sample group and all enrollees (data not shown). The database contains medical information regarding ambulatory care, inpatient care, and prescription drugs. Diagnoses were coded according to the International Classification of Disease, 9th revision (ICD-9) which was incorporated into the database since 2000 . The database used in this study can be interlinked by the scrambled unique individual's personal identification number (PIN). The NHRI safeguards the privacy and confidentiality of all beneficiaries and transfers the health insurance data to health researchers after ethical approval has been obtained. In this analysis, access to the NHIRD has been approved by the NHRI Ethics Review Committee.

Study population and end-point. Four groups of patients were included in our analysis: prostate cancer with ADT only, BPH with alpha-blockers only, BPH with 5-alpha reductase inhibitors (5ARIs), and healthy controls. The patient selection, sampling and exclusion criteria are shown in Figure 1. Inclusion criteria in the prostate cancer study group met two conditions: (i) newly diagnosed prostate cancer between 2001 and 2007; and (ii) ADT treatment. The exclusion criteria in the study group were: (i) any interventional prostate surgeries or radiation therapy prior to inclusion or after inclusion; (ii) chemotherapy or targeted therapy; (iii) medications for $\mathrm{OAB}$ for more than 1 month or $\mathrm{OAB}$ diagnosis before prostate cancer diagnosis; (iv) existence of neurological diseases requiring 3 or more visits to outpatient clinics or 1 or more inpatient visits and (v) other types of cancer. The diagnosis of prostate cancer was defined as code ICD-9-CM 185.0. Neurologic diseases were coded ICD-9-CM from 320 to 359 including the majority of central or peripheral neurological diseases. ADT was defined as luteinizing hormone release hormone (LH-RH) agonists or antagonists and other types of anti-androgens. Chemotherapy or targeted therapies were identified by drug scientific names. Medications for OAB were trospium, imipramine, flavoxate, propiverine, oxybutynin, tolterodine and solifenacin. In the BPH group, subjects who met the following criteria were also excluded: (i) receiving interventional prostate surgeries; (ii) previous $\mathrm{OAB}$ history; (iii) any kinds of neurologic diseases coded ICD-9-CM from 320 to 359; Incident cases of lower urinary tract symptoms were identified from the NHIRD after the upstream patient selection. The ratio of the number of subjects in the healthy control group to the number of prostate cancer patients was $2: 1$.

In all four groups, comorbidities, including myocardial infarction (MI, ICD-9-CM 410), heart failure (ICD-9-CM 428), peripheral vascular disease (PVD, ICD-9-CM 443), cerebrovascular accident (CVA, ICD-9-CM 434), dementia (ICD-9-CM 290-294), chronic obstructive pulmonary disease (COPD, ICD-9-CM 490-496), mild chronic liver disease except cirrhosis (CLD, ICD-9-CM 571, except $571.2,571.5,571.6$ ), chronic kidney disease (CKD; ICD-9-CM 582-583), diabetes mellitus (DM, ICD-9-CM 250), hyperthyroidism (ICD-9-CM 410-414) were recorded.
Statistical analysis. The data are presented as the mean values and standard deviations (SD) for continuous variables, and proportions for categorical variables. The differences between continuous values were analyzed by using $t$ test for continuous variables, and chisquare test for categorical variables. Multivariate Cox proportional hazard regression was used to estimate the hazard ratio (HR) and 95\% confidence interval (CI) for the association between the prevalence of $\mathrm{OAB}$ and the four divided groups. Propensity analysis was used for further confirming this association. The cumulative incidence curves were plotted via the Kaplan-Meier method with statistical significance examined by the log-rank test. All statistical analyses were carried out by SAS software version 9.2 (SAS Institute, Inc., Cary, NC, USA). A $p$-value of $<0.05$ indicated statistically significant differences.

\section{Results}

There were 19,674 subjects who met the primary inclusion criteria in the study group. After elimination of subjects based on exclusion criteria. 2,629 cases were selected as study subjects. The control group included 484,259 subjects selected randomly from a 1 million non-cancer population. Among them, 415,322 subjects were selected as healthy controls without BPH. After 1:2 match with study subjects, 5258 subjects were selected as healthy controls. The BPHalpha blocker included 14,151 individuals who received alpha-blockers as principal treatment without any 5ARI. The BPH-5ARI group included 1,056 subjects who received medications of 5ARI as principal treatment with or without any alpha-blockers.

Table I which compares the ages and comorbidities of the four groups shows that the prostate cancer group had an older age $(p<0.001)$ than the other $2 \mathrm{BPH}$ groups, but not the healthy controls. The mean age of the prostate cancer cohort was 73.8 years at the time of the survey. A comparison of comorbidities shows a variation among groups. The prostate cancer group had a higher proportion of $\mathrm{MI}(p<0.001)$, heart failure $(p<0.001)$, CVA $(p<0.001)$, COPD $(p<0.001)$, CLD $(p<0.001)$, CKD $(p<0.001)$ and DM $(p<0.001)$ than the healthy control group. The healthy controls had a higher proportion of hyperthyroidism $(p<0.001)$ than the prostate cancer group. In regard to PVD and dementia, there was no statistically significant difference between the two groups ( $p=0.11$ and $p=0.41$ respectively). The prostate cancer group also had higher proportion of all the chronic disease variables except hyperthyroidism than the BPH-alpha blocker group. However, the prostate cancer group and the BPH-5ARI group were similar in MI, PVD, CVA, dementia, CKD, and DM. The prostate cancer group had a higher proportion of heart failure and COPD ( $p=0.002$ and $p=0.04$ respectively), while the BPH-5ARI group had a higher proportion of CLD and hyperthyroidism ( $p=0.003$ and $p<0.0001$ respectively).

In the prostate cancer group $109 \mathrm{OAB}$ cases were identified with the rate of 56.6 per 10,000 person-years compared to only 3 cases in the healthy control group $(0.85$ per 10,000 


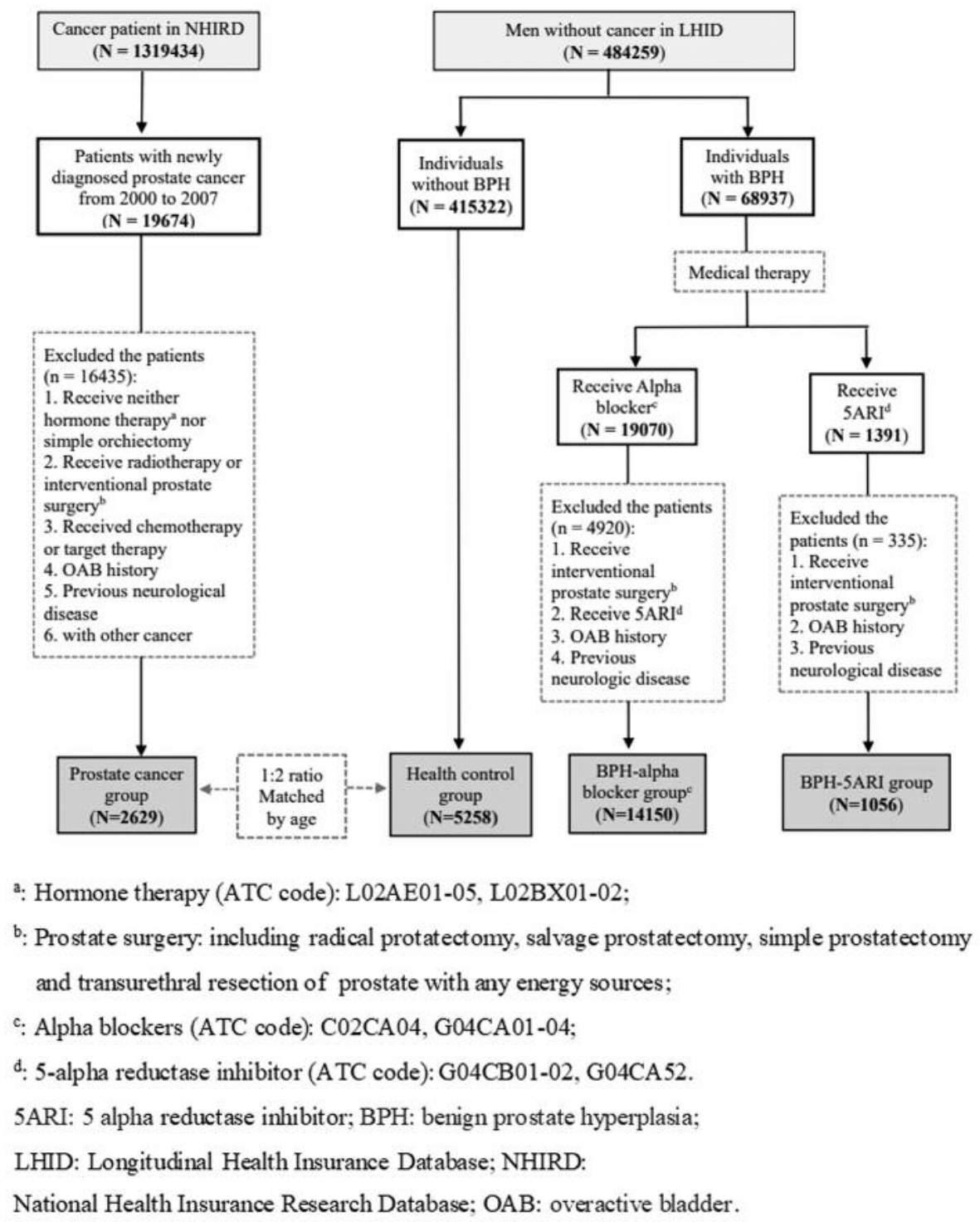

Figure 1. Flow diagram showing the process of overactive bladder $(O A B)$ patient sampling and participation.

person-years, Table II). The crude model showed a significant decreased risk of $\mathrm{OAB}$ in the $\mathrm{BPH}$-alpha-blocker $(\mathrm{HR}=0.57$, $95 \% \mathrm{CI}=0.46-0.7)$ and in the healthy control group $(\mathrm{HR}=0.01$, $95 \% \mathrm{CI}=0.005-0.05)$ compared to the prostate cancer group. After adjustment for comorbidities, the OAB risk was still lower in both groups $(\mathrm{HR}=0.7,95 \% \mathrm{CI}=0.56-0.87, \mathrm{HR}=0.02$, $95 \% \mathrm{CI}=0.01-0.05$ respectively) compared to the prostate cancer group. However, the BPH-5ARI group showed a significantly higher risk of $\mathrm{OAB}$ (adjusted $\mathrm{HR}=1.49$, $95 \% \mathrm{CI}=1.08-2.06)$ compared with the prostate cancer group.

In a subgroup analysis, the healthy control group exhibited a low $\mathrm{OAB}$ risk in both age groups $(0$ in the age $<65$ years and $\mathrm{HR}=0.02,95 \% \mathrm{CI}=0.56-0.87$, in the age $\geq 65$ years $)$. OAB risk was also lower in the healthy control group with $(\mathrm{HR}=0.01$, $95 \% \mathrm{CI}=0.001-0.07)$ and without comorbidities $(\mathrm{HR}=0.02$, 95\% CI $=0.01-0.09$ ) (Table III). Compared to the prostate cancer group, the BPH-alpha blocker group showed no difference in $\mathrm{OAB}$ risks below age $65(\mathrm{HR}=0.64,95 \% \mathrm{CI}=0.35-1.18)$ or without comorbidities $(\mathrm{HR}=0.69,95 \% \mathrm{CI}=0.46-1.03)$ (Table III). The risk decreased in the BPH-alpha blocker group at ages above $65(\mathrm{HR}=0.68,95 \% \mathrm{CI}=0.54-0.87)$ and in the subgroup with comorbidities $(\mathrm{HR}=0.7,95 \% \mathrm{CI}=0.54-0.91)$ (Table III). However, the BPH-5ARI group exhibited a higher risk of OAB at ages above $65(\mathrm{HR}=1.5,95 \% \mathrm{CI}=1.02-2.2)$ or with comorbidities $(\mathrm{HR}=1.58,95 \% \mathrm{CI}=1.08-2.3)$ compared with the prostate cancer group. 
Table I. Baseline variables among the four groups.

\begin{tabular}{|c|c|c|c|c|c|c|c|}
\hline \multirow[t]{2}{*}{ Variable } & \multirow{2}{*}{$\begin{array}{l}\text { Prostate cancer } \\
\quad \mathrm{N}=2,629\end{array}$} & \multirow{2}{*}{$\begin{array}{l}\text { BPH-alpha blocker } \\
\qquad \mathrm{N}=14,150\end{array}$} & \multirow{2}{*}{$\begin{array}{l}\mathrm{BPH}-5 \mathrm{ARI} \\
\mathrm{N}=1,056\end{array}$} & \multirow{2}{*}{$\begin{array}{l}\text { Health control } \\
\mathrm{N}=5,258\end{array}$} & \multicolumn{3}{|c|}{$p$-Value } \\
\hline & & & & & $\begin{array}{c}\text { Prostate cancer } \\
v s . \text { healthy control }\end{array}$ & $\begin{array}{c}\text { Prostate cancer } \\
v s . \mathrm{BPH} \text {-alpha blocker }\end{array}$ & $\begin{array}{r}\text { Prostate cancer } \\
v s . \text { BPH-5ARI }\end{array}$ \\
\hline Age group (years)* & $73.8(7.77)$ & $62.3(11.3)$ & $66.3(9.91)$ & $73.5(7.96)$ & 0.26 & $<0.0001$ & $<0.0001$ \\
\hline$<45$ & $2(0.08)$ & $720(5.09)$ & $9(0.85)$ & $4(0.08)$ & & & \\
\hline $45-64$ & $309(11.8)$ & $7,270(51.4)$ & $450(42.6)$ & $618(11.8)$ & & & \\
\hline$\geq 65$ & $2,318(88.2)$ & $6,160(43.5)$ & $597(56.5)$ & $4636(88.2)$ & & & \\
\hline \multicolumn{8}{|l|}{ Comorbidity } \\
\hline MI & $96(3.65)$ & $304(2.15)$ & $32(3.03)$ & $147(2.80)$ & 0.04 & $<0.0001$ & 0.35 \\
\hline Heart failure & $248(9.43)$ & $716(5.06)$ & $67(6.34)$ & $384(7.30)$ & 0.001 & $<0.0001$ & 0.002 \\
\hline PVD & $73(2.78)$ & $260(1.84)$ & $30(2.84)$ & $115(2.19)$ & 0.11 & 0.002 & 0.91 \\
\hline CVA & $581(22.1)$ & $2,198(15.5)$ & $222(21.0)$ & $970(18.5)$ & 0.0001 & $<0.0001$ & 0.47 \\
\hline Dementia & $77(2.93)$ & $224(1.58)$ & $24(2.27)$ & $172(3.27)$ & 0.41 & $<0.0001$ & 0.27 \\
\hline COPD & $1,172(44.6)$ & $4,817(34.0)$ & $432(40.9)$ & $1,621(30.8)$ & $<0.0001$ & $<0.0001$ & 0.04 \\
\hline CLD & $431(16.4)$ & $2,724(19.3)$ & $217(20.6)$ & $464(8.82)$ & $<0.0001$ & 0.0006 & 0.003 \\
\hline CKD & $63(2.40)$ & $197(1.39)$ & $17(1.61)$ & $60(1.14)$ & $<0.0001$ & 0.0001 & 0.14 \\
\hline $\mathrm{DM}$ & $547(20.8)$ & $2611(18.5)$ & $234(22.2)$ & $829(15.8)$ & $<0.0001$ & 0.005 & 0.36 \\
\hline Hyperthyroidism & 0 & $87(0.61)$ & $11(1.04)$ & $20(0.38)$ & 0.001 & $<0.0001$ & $<0.0001$ \\
\hline
\end{tabular}

MI: Myocardial infarct; PVD: peripheral vascular disease; CVA: cerebrovascular accident; COPD: chronic obstructive pulmonary disease; CLD: chronic liver disease; CKD: chronic kidney disease; DM: diabetes mellitus.

Figure 2 shows a time-dependent increase in the cumulative incidence of $\mathrm{OAB}$ among the prostate cancer, $\mathrm{BPH}$-alpha blocker and the BPH-5ARI groups (Log-Rank test, $p<0.0001)$.

\section{Discussion}

Our study indicated that androgen ablation treatment is associated with an increased risk of $\mathrm{OAB}$ and that an increase in treatment duration increases cumulative risk of $\mathrm{OAB}$. We also found some interesting points from this cohort study. First, androgen ablation therapy in prostate cancer patients eventually generated $\mathrm{OAB}$ incidence that equaled the incidence of $\mathrm{OAB}$ in $\mathrm{BPH}$. Second, the rate of $\mathrm{OAB}$ in the prostate cancer group seemed to increase with age similarly to the increase observed in both $\mathrm{BPH}$ groups. Below the age of 65 , the risk of OAB did not differ between prostate cancer group and both BPH groups, suggesting that the younger age may tolerate more structural changes. Third, the prominent delay in the development of OAB after the start of ADT possibly also indicates slowly developing structural changes in the lower urinary tract, rather than rapid physiological changes that have been demonstrated after castration in animals (8).

Since ADT is still the pivotal treatment in advanced prostate cancer, OAB which might occur after ADT should be taken into consideration during patient care. ADT alone has been shown to increase osteoporosis and possibility of fracture while $\mathrm{OAB}$ may also increase the risk of fracture in the elderly $(10,11)$.

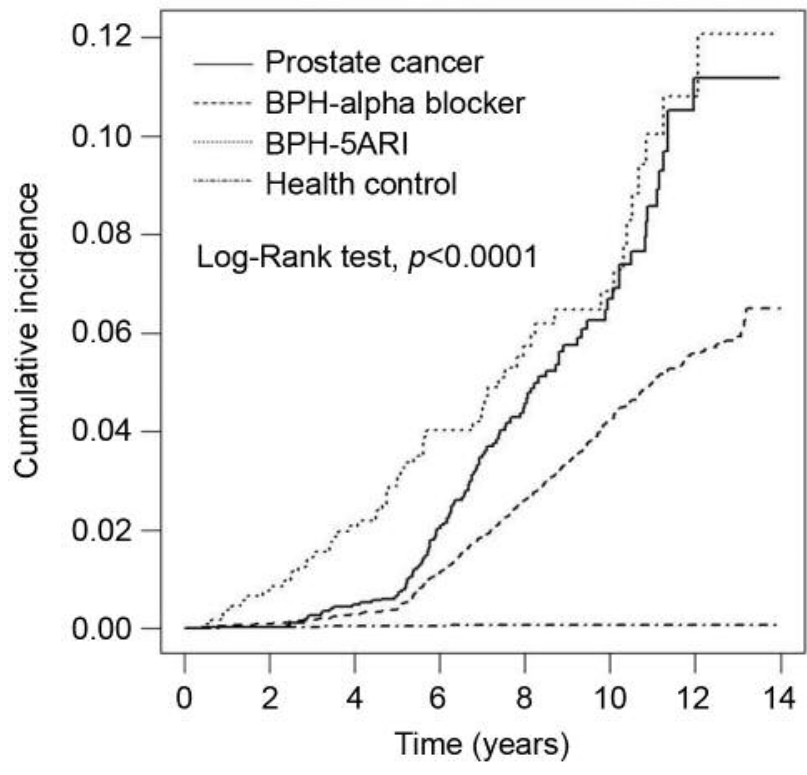

Figure 2. Kaplan-Meier cumulative incidence of developing $O A B$ among four groups $(p<0.0001$, log-rank test) $(n=23,093)$.

Our findings also corresponded to previous epidemiological studies. Kopp et al. performed a cross-sectional study of 5990 fracture patients and found that ADT was associated with increased risk of urinary incontinence which also rose with increasing treatment duration (12). Carlsson et al. used a nationwide population-based study in the evaluation of long- 
Table II. Multi-variant analysis of OAB risk.

\begin{tabular}{|c|c|c|c|c|c|}
\hline Variables & Event & Patient-Years & Rate & Model $1(95 \% \mathrm{CI})$ & Model $2(95 \% \mathrm{CI})$ \\
\hline \multicolumn{6}{|l|}{ Treatment } \\
\hline Prostate cancer & 109 & 19,260 & 56.6 & ref & ref \\
\hline BPH-alpha-blocker & 501 & 128,644 & 38.9 & $0.57(0.46-0.70)$ & $0.70(0.56-0.87)$ \\
\hline BPH-5ARI & 58 & 7,714 & 75.2 & $1.29(0.94-1.77)$ & $1.49(1.08-2.06)$ \\
\hline Health control & 3 & 35,226 & 0.85 & $0.01(0.005-0.05)$ & $0.02(0.01-0.05)$ \\
\hline Age, years & - & - & - & $1.01(1.01-1.02)$ & $1.02(1.01-1.02)$ \\
\hline \multicolumn{6}{|l|}{ MI } \\
\hline No & 655 & 186,710 & 35.1 & ref & ref \\
\hline Yes & 16 & 4,133 & 38.7 & $1.22(0.74-2.00)$ & $0.92(0.55-1.52)$ \\
\hline \multicolumn{6}{|l|}{ Heart failure } \\
\hline No & 628 & 181,264 & 34.7 & ref & ref \\
\hline Yes & 43 & 9,579 & 44.9 & $1.49(1.09-2.03)$ & $1.14(0.83-1.57)$ \\
\hline \multicolumn{6}{|l|}{ PVD } \\
\hline No & 656 & 187,443 & 35.0 & ref & ref \\
\hline Yes & 15 & 3,400 & 44.1 & $1.39(0.83-2.32)$ & $1.09(0.65-1.82)$ \\
\hline \multicolumn{6}{|l|}{ CVA } \\
\hline No & 532 & 161,900 & 32.9 & ref & ref \\
\hline Yes & 139 & 28,944 & 48.0 & $1.63(1.35-1.96)$ & $1.31(1.08-1.59)$ \\
\hline \multicolumn{6}{|l|}{ Dementia } \\
\hline No & 655 & 187,805 & 34.9 & ref & ref \\
\hline Yes & 16 & 3,038 & 52.7 & $1.83(1.12-3.01)$ & $1.30(0.78-2.15)$ \\
\hline \multicolumn{6}{|l|}{ COPD } \\
\hline No & 400 & 128,337 & 31.2 & ref & ref \\
\hline Yes & 271 & 62,506 & 43.4 & $1.49(1.28-1.74)$ & $1.19(1.02-1.40)$ \\
\hline \multicolumn{6}{|l|}{ CLD } \\
\hline No & 534 & 159,623 & 33.5 & ref & ref \\
\hline Yes & 137 & 31,221 & 43.9 & $1.36(1.13-1.64)$ & $1.24(1.02-1.50)$ \\
\hline \multicolumn{6}{|l|}{ CKD } \\
\hline No & 658 & 188,297 & 34.9 & ref & ref \\
\hline Yes & 13 & 2,546 & 51.1 & $1.58(0.91-2.74)$ & $1.29(0.74-2.24)$ \\
\hline \multicolumn{6}{|l|}{ DM } \\
\hline No & 540 & 158,415 & 34.1 & ref & ref \\
\hline Yes & 131 & 32,429 & 40.4 & $1.28(1.05-1.55)$ & $1.08(0.89-1.31)$ \\
\hline \multicolumn{6}{|l|}{ Hyperthyroidism } \\
\hline No & 668 & 189,877 & 35.2 & ref & ref \\
\hline Yes & 3 & 967 & 31.0 & $0.87(0.28-2.72)$ & $0.85(0.27-2.65)$ \\
\hline
\end{tabular}

Model 1: Crude model. Model 2: Adjusted for age, MI, heart failure, PVD, CVA, dementia, COPD, CLD, CKD, DM and hyperthyroidism. MI: Myocardial infarct; PVD: peripheral vascular disease; CVA: cerebrovascular accident; COPD: chronic obstructive pulmonary disease; CLD: chronic liver disease; CKD: chronic kidney disease; DM: diabetes mellitus.

Table III. Subgroup analysis of four groups per 10,000 person-years.

\begin{tabular}{|c|c|c|c|c|c|c|c|c|}
\hline \multirow[b]{2}{*}{ Variables } & \multicolumn{2}{|c|}{ Prostate cancer } & \multicolumn{2}{|c|}{ BPH-alpha blocker } & \multicolumn{2}{|c|}{ BPH-5ARI } & \multicolumn{2}{|c|}{ Health control } \\
\hline & Rate & HR $(95 \% \mathrm{CI})$ & Rate & HR (95\%CI) & Rate & HR (95\%CI) & Rate & HR (95\%CI) \\
\hline \multicolumn{9}{|c|}{ Age groups* } \\
\hline$<65$ & 44.7 & ref & 33.3 & $0.64(0.35-1.18)$ & 61.5 & $1.35(0.65-2.78)$ & 0 & - \\
\hline$\geq 65$ & 58.3 & ref & 46.9 & $0.68(0.54-0.87)$ & 87.0 & $1.50(1.02-2.20)$ & 1.01 & $0.02(0.01-0.06)$ \\
\hline \multicolumn{9}{|c|}{ Comorbidity** } \\
\hline No & 53.8 & ref & 32.7 & $0.69(0.46-1.03)$ & 55.9 & $1.21(0.64-2.29)$ & 0.53 & $0.01(0.001-0.07)$ \\
\hline Yes & 57.9 & ref & 43.2 & $0.70(0.54-0.91)$ & 84.5 & $1.58(1.08-2.30)$ & 1.21 & $0.02(0.01-0.09)$ \\
\hline
\end{tabular}

* Model adjusted for age, myocardial infarct, heart failure, peripheral vascular disease, stroke, dementia, chronic obstructive pulmonary disease, chronic liver disease, chronic kidney disease, diabetes mellitus and hyperthyroidism. ** Model adjusted for age. 
term functional outcomes among prostate cancer patients regardless of treatment duration association. ADT treatment alone also was associated with increased risk of urinary incontinence compared with active surveillance (13).

However, because of the different methods used in the present study, there are some differences from those previous studies. For example, two other Asian prospective studies showed an improvement of lower urinary tract symptoms (LUTS) in prostate cancer patients who received ADT (14, 15). Their finding focused on prostate volume decrease, urodynamic parameters and improvement of voiding scores while the storage symptom scores were not different. The mean follow-up periods were 2 years or less and the sample sizes were less than 200 subjects.

In order to clarify the impact of male LUTS in the general population in our study, BPH patients were included for comparison with the prostate cancer group. The BPH-alpha blocker group still showed a decreased risk in $\mathrm{OAB}$ $(\mathrm{HR}=0.7,95 \% \mathrm{CI}=0.56-0.87)$ after modification for age and chronic comorbidities. Interestingly, BPH-5ARI seemed to be associated with a higher risk of $\mathrm{OAB}$ than prostate cancer. This was quite reasonable in this database setting since the prostate size must be larger than $30 \mathrm{ml}$ or the maximal voiding flow must be smaller than $15 \mathrm{ml} / \mathrm{min}$ according to the reimbursement regulation. With this regulation, these patients should have more severe LUTS than the other 3 groups. This is an important point, since previous studies found the $50 \%$ coexistence rate of bladder outlet obstruction and $\mathrm{OAB}(7,16,17)$. In Figure 2, both the $\mathrm{BPH}$ groups showed a parallel time-dependent increase of OAB incidence which implies a similar potential of the two groups. The prostate cancer study group showed a significant rise of the cumulative incidence speed after 5 years of ADT treatments. This phenomenon suggests ADT accelerates the rate of $\mathrm{OAB}$ formation after treatment for 5 years.

This study has several limitations. First, the definition of $\mathrm{OAB}$ was based on consecutive drug prescriptions for treatment of $\mathrm{OAB}$ for a period more than one month instead on the diagnosis of $\mathrm{OAB}$. Although this method was more relevant in clinical situations in terms of physicians' habits and insurance regulations, it included patients who had only storage symptoms such as frequency and nocturia, not OAB. Second, in order to eliminate the impact of neurologic diseases and anatomic influence to the lower urinary tract after local therapies, all subjects who were diagnosed with neurological disorders or had been submitted to procedures mentioned in the database were excluded. This resulted in censored OAB subject reduction to only 3 in the healthy control group decreasing the power of this study. Third, our study group included prostate cancer patients who were easier to seek medical service than healthy subjects. Previous epidemiological studies all showed that a quite low percentage of OAB patients seek medical services. This may have influenced the result of this study.

\section{Conclusion}

Our study disclosed that ADT was associated with an increased risk of $\mathrm{OAB}$ in prostate cancer patients. This result explained the role of androgen in maintenance of male voiding threshold and highlighted de novo development of $\mathrm{OAB}$ after ADT which may influence quality of life in the elderly prostate cancer patients.

\section{Conflicts of Interest}

The Authors declare no competing financial interests.

\section{Acknowledgements}

This study is based, in part, on data obtained from the National Health Insurance Research Database provided by the Bureau of National Health Insurance, Department of Health, Taiwan, and managed by the National Health Research Institutes. The interpretation and conclusions contained herein do not represent those of the Bureau of National Health Insurance, Department of Health, or National Health Research Institutes, Taiwan.

\section{References}

1 Chavalmane AK, Comeglio P, Morelli A, Filippi S, Fibbi B, Vignozzi L, Sarchielli E, Marchetta M, Failli P, Sandner P, Saad F, Gacci M, Vannelli GB and Maggi M: Sex steroid receptors in male human bladder: expression and biological function. J Sex Med 7: 2698-2713, 2010.

2 Miyamoto H, Yao JL, Chaux A, Zheng Y, Hsu I, Izumi K, Chang C, Messing EM, Netto GJ and Yeh S: Expression of androgen and oestrogen receptors and its prognostic significance in urothelial neoplasm of the urinary bladder. BJU Int 109: 1716-1726, 2012.

3 Badawi JK, Bosch R, Djurhuus JC and Hanna-Mitchell AT: Is testosterone important in LUT function in men and women? ICIRS 2015. Neurourol Urodyn 36: 859-862, 2017.

4 Cheng CL and de Groat WC: Effects of agonists for estrogen receptor alpha and beta on ovariectomy-induced lower urinary tract dysfunction in the rat. Am J Physiol Renal Physiol 306: F181-187, 2014.

5 Cheng CL, Li JR, Lin $\mathrm{CH}$ and de Groat WC: Positive association of female overactive bladder symptoms and estrogen deprivation: A nationwide population-based cohort study in Taiwan. Medicine 95: e4107, 2016.

6 Irwin DE, Milsom I, Kopp Z, Abrams P, Artibani W and Herschorn S: Prevalence, severity, and symptom bother of lower urinary tract symptoms among men in the EPIC study: impact of overactive bladder. Eur Urol 56: 14-20, 2009.

7 Coyne KS, Sexton CC, Bell JA, Thompson CL, Dmochowski R, Bavendam T, Chen CI and Quentin Clemens J: The prevalence of lower urinary tract symptoms (LUTS) and overactive bladder $(\mathrm{OAB})$ by racial/ethnic group and age: results from OAB-POLL. Neurourol Urodyn 32: 230-237, 2013.

8 Cheng CL and de Groat WC: Effect of orchiectomy and testosterone replacement on lower urinary tract function in anesthetized rats. Am J Physiol Renal Physiol 311: F864-f70, 2016. 
9 Hristov KL, Parajuli SP, Provence A and Petkov GV Testosterone decreases urinary bladder smooth muscle excitability via novel signaling mechanism involving direct activation of the BK channels. Am J Physiol Renal Physiol 311: F1253-f9, 2016.

10 Wagner TH, Hu TW, Bentkover J, LeBlanc K, Stewart W, Corey $\mathrm{R}$, Zhou $\mathrm{Z}$ and Hunt $\mathrm{T}$ : Health-related consequences of overactive bladder. Am J Manag Care 8: S598-607, 2002.

11 Karabulut A, Simavli S, Demirtas O, Ok N, Gungor HR and Zumrutbas A: Evaluation of overactive bladder and nocturia as a risk factor for hip fracture in climacteric women: a matched pair case control study. J Obstet Gynaecol 38: 252-256, 2018.

12 Kopp RP, Marshall LM, Wang PY, Bauer DC, Barrett-Connor E and Parsons JK: The burden of urinary incontinence and urinary bother among elderly prostate cancer survivors. Eur Urol 64: 672-679, 2013.

13 Carlsson S, Drevin L, Loeb S, Widmark A, Lissbrant IF, Robinson D, Johansson E, Stattin P and Fransson P: Populationbased study of long-term functional outcomes after prostate cancer treatment. BJU Int 117: E36-45, 2016.
14 Washino S, Hirai M, Saito K, Kobayashi Y, Arai Y and Miyagawa T: Impact of androgen deprivation therapy on volume reduction and lower urinary tract symptoms in patients with prostate cancer. Low Urin Tract Symptoms 10: 57-63, 2018.

15 Choi H, Chung H, Park JY, Lee JG and Bae JH: the influence of androgen deprivation therapy on prostate size and voiding symptoms in prostate cancer patients in Korea. Int Neurourol J 20: 342-348, 2016.

16 Chung DE and Sandhu JS: Overactive bladder and outlet obstruction in men. Curr Urol Rep 12: 77-85, 2011.

17 Chapple CR and Roehrborn CG: A shifted paradigm for the further understanding, evaluation, and treatment of lower urinary tract symptoms in men: focus on the bladder. Eur Urol 49: 651$658,2006$.
Received October 8, 2018

Revised November 21, 2018

Accepted November 22, 2018 